\title{
The resonance behavior for the coupling of two Aw-Rascle traffic models
}

\author{
Yanbo Hu ${ }^{1}$ and Guodong Wang ${ }^{2 *}$
}

"Correspondence:

gdwang@ahjzu.edu.cn

${ }^{2}$ School of Mathematics and

Physics, Anhui Jianzhu University, Hefei, P.R. China

Full list of author information is

available at the end of the article

\section{Springer}

\begin{abstract}
By studying the Riemann problem for the Aw-Rascle traffic model with different pressure laws, which is the coupling of two one-dimensional hyperbolic systems, we investigate the resonance phenomena. The main difficulty arises from the possible resonance behavior which may result in multiple solutions. We discover a new and interesting phenomenon showing that there exist infinitely many solutions for some certain initial data, which is quite different compared to earlier studies for the isentropic model of a fluid flow in a nozzle with variable cross-section and the shallow water equations with discontinuous topography. In order to overcome this difficulty, we impose the so-called TV-condition to obtain the uniqueness of solution to the Riemann problem.
\end{abstract}

MSC: 35L65; 35J70; 35R35

Keywords: Aw-Rascle model; Different pressure laws; Riemann problem; Resonance; TV-condition

\section{Introduction}

Resonance phenomenon, occurring in many hyperbolic equations, is an important and interesting phenomenon in both physical and mathematical sense and has attracted the attention of many researchers. When one of the nonlinear wave families of the hyperbolic equations has a zero speed wave, the resonance behavior may result in multiple solutions to the certain initial value problem [23, 24, 30, 31]. For more related results, see e.g. [14, 17]. It is therefore natural to add some admissibility criteria to single out the physically relevant solution. However, until now, this is still an open problem.

In the last decades, many efforts have been made to study the resonance behavior for specific hyperbolic equations such as systems of balance laws in non-conservation laws and the coupled Euler systems with distinct pressure laws. In particular, when they investigated the Riemann problems for the non-conservation laws of the isentropic flows in a nozzle with variable cross-section and the shallow water equations with discontinuous topography, LeFloch and Thanh [27-29] introduced an admissibility criterion, called monotonicity criterion (i.e., along any stationary curve, the bottom level $a$ is monotone as a function of $h$ and the total variation of the bottom level component of any Riemann solution must not exceed $\left.\left|a_{L}-a_{R}\right|\right)$, to provide the existence and uniqueness of Riemann solutions

(c) The Author(s) 2020. This article is licensed under a Creative Commons Attribution 4.0 International License, which permits use, sharing, adaptation, distribution and reproduction in any medium or format, as long as you give appropriate credit to the original author(s) and the source, provide a link to the Creative Commons licence, and indicate if changes were made. The images or other third party material in this article are included in the article's Creative Commons licence, unless indicated otherwise in a credit line to the material. If material is not included in the article's Creative Commons licence and your intended use is not permitted by statutory regulation or exceeds the permitted use, you will need to obtain permission directly from the copyright holder. To view a copy of this licence, visit http://creativecommons.org/licenses/by/4.0/ 
in the nonresonant regime and multiple solutions in the resonant regime. In [6], Andrianov and Warnecke considered the Riemann problem for the compressible duct flow and applied the so-called evolutionarity criterion to rule out the nonphysical solutions. Moreover, based on the 2D numerical results, they discovered that the physically relevant $1 \mathrm{D}$ solutions satisfy a kind of entropy rate admissibility criterion. Thanh [37] used the monotonicity criterion to discuss the Riemann problem for the above compressible duct flow again and established the existence for large data and uniqueness in strictly hyperbolic domains. By means of the monotonicity criterion, he [38] also established the monotonicity of the curves of composite waves for the shallow water equations with discontinuous topography, which provides an important method to discover the domain of uniqueness and a deterministic version of the existence of Riemann solutions. In addition, Marchesin and Pase-Leme [32] defined 1-M and 2-M curves to construct Riemann solutions for the $2 \times 2$ isothermal gas dynamics in a duct with discontinuous diameter. They found that there exist multiple solutions for the Riemann problem with some certain initial data due to the occurrence of the bifurcation phenomena. Following the work in [32], Han, Hantkee, and Warnecke [18-20] developed the two curves 1-M and 2-M and analyzed the Riemann problem for the compressible duct flow and the shallow water equations with discontinuous topography.

To the best of our knowledge, many criteria have been proposed for investigating the resonance behavior to different hyperbolic models, and a unique solution among the possibly multiple solutions has not been picked out. The main purpose of this paper is designed to explore this problem through a discussion of the Riemann problem for the well-known Aw-Rascle traffic model with distinct pressure laws, which can be seen as an attempt to study the coupling of two one-dimensional barotropic Euler systems at an interface at $x=0$ in [1] and [33]. We can see [8-10] about resonance behavior for the corresponding coupling of two different hyperbolic systems. We also refer the reader to [5, 12, 13, 26, 29, 39] and the references therein for the numerical methods to study the related hyperbolic systems.

The Aw-Rascle traffic model with distinct pressure laws reads as follows:

$$
\left\{\begin{array}{l}
\rho_{t}+(\rho u)_{x}=0, \\
(\rho(u+p))_{t}+(\rho u(u+p))_{x}=0,
\end{array}\right.
$$

where

$$
p(\rho, x)=(1-H(x)) p_{L}(\rho)+H(x) p_{R}
$$

$p_{\alpha}=\kappa_{\alpha} \rho^{\gamma}$ denote some kind of pressure, $\alpha=L, R$, and $H(x)$ is the Heaviside function and $\kappa_{\alpha}$ denotes the road condition. If $\kappa_{L}=\kappa_{R}$, then system (1.1)-(1.2) becomes the classical Aw-Rascle traffic model, which was introduced by Aw and Rascle [7] and has been widely studied by many authors, see $[1,25,34]$ among others.

System (1.1)-(1.2) with $\kappa_{L} \neq \kappa_{R}$ is a model of coupling. In this case, the system can be considered as the Aw-Rascle traffic model with the rough road condition. We would like to emphasize that the coupling systems are not easy to solve, even for the Riemann problems. The main reason is that the non-uniqueness of solution is related to the resonance that may take place at the interface $x=0$ and the coupling condition must be chosen to achieve two 
well-posed initial boundary value problems in both sides of $x=0[4,8,9]$. It is worthwhile to mention the work by Ambroso et al. [1-4] who used a relaxation method to seek for a conservative coupling which avoided resonance.

In order to solve the Riemann problem for (1.1)-(1.2), we supplement the system with the trivial equation

$$
\kappa_{t}=0 .
$$

Then the problem is changed into

$$
\left\{\begin{array}{l}
\rho_{t}+(\rho u)_{x}=0, \\
(\rho(u+p))_{t}+(\rho u(u+p))_{x}=0, \\
\kappa_{t}=0,
\end{array}\right.
$$

with the piecewise constant initial data

$$
U_{0}=(\rho, u, \kappa)(x, 0)= \begin{cases}\left(\rho_{L}, u_{L}, \kappa_{L}\right), & x<0 \\ \left(\rho_{R}, u_{R}, \kappa_{R}\right), & x>0 .\end{cases}
$$

By the Aw-Rascle traffic model, we require that $\rho(x, t) \geq 0$ and velocity $u(x, t) \geq 0$ of cars at $(x, t)$. Furthermore, in this paper we assume $\kappa_{\alpha}>1, \alpha=L, R$, and $1<\gamma<2$.

Comparing with the Riemann problems for the isentropic model of a fluid flow in a nozzle with variable cross-section and the shallow water equations with discontinuous topography dealt with by LeFloch and Thanh [27-29], there are two important differences which are the main problems that we will solve in this paper. The first one is that the resonance hypersurfaces on both sides of the interface $x=0$ are not the same, which makes the construction of the Riemann solution more difficult. Most importantly, for given $U_{L}=$ $\left(\rho_{L}, u_{L}, \kappa_{L}\right)$, there is usually $U_{L}^{\sharp 0} \neq U_{L}^{0 \sharp}$ for the above two models, while we here have $U_{L}^{\sharp 0}=$ $U_{L}^{0 \sharp}$ (see Lemma 4.2), which results in system (1.4)-(1.5) having infinitely many solutions for certain Riemann data (see subcases (A5) or (B5)).

In order to overtake this difficulty, following the line of $[15,16,21,22]$, we propose the so-called TV-condition which allows us to single out a unique solution among possibly infinitely many solutions. Denote $\mathcal{W}_{0}=\mathcal{W}_{0}\left(U_{L}, U_{R}\right), W_{0}^{i}=W_{0}^{i}\left(U_{L}^{i}, U_{R}^{i}\right)$, and the total variation of the variable $\rho$ along all the stationary contact wave curves $\mathcal{W}_{0}=\sum_{i} W_{0}^{i}$ by $\operatorname{TV}_{W_{0}}(\rho)=\sum_{i}\left|\rho_{r}^{i}-\rho_{l}^{i}\right|$ across the discontinuity $x=0$, where $W_{0}^{i}$ denotes the $i$ th stationary contact wave connecting the left-hand state $U_{L}^{i}=\left(\rho_{L}^{i}, u_{L}^{i}, \kappa_{L}^{i}\right)$ and the right-hand state $U_{R}^{i}=\left(\rho_{R}^{i}, u_{R}^{i}, \kappa_{R}^{i}\right)$. Through our analysis, $\rho_{l}^{i}$ and $\rho_{r}^{i}$ may not be unique. To obtain the unique Riemann solution, across the stationary contact wave curve $W_{0}^{i}$, we select $\rho_{l}^{i}$ and $\rho_{r}^{i}$ such that

$$
\underset{W_{0}}{\operatorname{TV}}(\rho)=\sum_{i}\left|\rho_{r}^{i}-\rho_{l}^{i}\right| \quad \text { is minimum, }
$$

which is called the $T V$-condition in this paper. We will see that our TV-condition is quite effective not only for dealing with the resonance phenomenon but also for selecting a unique admissibility solution among infinitely many solutions. 
In a recent paper [33], Shen studied system (1.4) by introducing two variables $(u, \omega)=$ $\left(u, u+\kappa \rho^{\gamma}\right)$ and then dividing the construction of the global Riemann solver into two steps. The last step is to solve a scalar conservation law with discontinuous flux under the minimum jump path cross the discontinuity $x=0$. The similar minimum jump condition was first introduced by Gimse and Risebro [16] to study the conservation laws with the discontinuous flux. The key difference with this paper and the paper [33] is whether the stationary contact discontinuity and the shock with zero speed occur. In this paper, there occur the stationary contact discontinuity and the shock with zero speed to some Riemann solutions constructed by the variables $(\rho, u, \kappa)$.

The main results of this paper can be demonstrated as follows.

Theorem 1.1 Given the left and right states $U_{L}=\left(\rho_{L}, u_{L}, \kappa_{L}\right)$ and $U_{R}=\left(\rho_{R}, u_{R}, \kappa_{R}\right)$, suppose that condition (3.10) is satisfied, where $\omega_{0}=\omega_{L}$ and $m_{0}=m_{L}$. If $T V$-condition (1.6) is carried out, then Riemann problem (1.4) and (1.5) has a unique solution.

The paper is organized as follows. In Sect. 2, we give the corresponding preliminaries for (1.4). In Sect. 3, we investigate the properties of stationary shock wave and stationary contact wave. In Sect. 4, we introduce the so-called TV-condition and then give two related lemmas. In Sect. 5, under the TV-condition, we construct the corresponding Riemann solution case by case. Moreover, we obtain the uniqueness of the Riemann solution, which is demonstrated in Theorem 1.1. Finally, the conclusions are presented.

\section{Preliminaries}

Setting the dependent variable $U=(\rho, u, \kappa)$, the Jacobian matrix of system (1.4) is in the form

$$
A(U)=\left(\begin{array}{ccc}
u & \rho & 0 \\
0 & u-\kappa \gamma \rho^{\gamma} & \rho^{\gamma} u \\
0 & 0 & 0
\end{array}\right)
$$

which has three eigenvalues

$$
\lambda_{0}=0, \quad \lambda_{1}=u-\kappa \gamma \rho^{\gamma}, \quad \lambda_{2}=u,
$$

together with the corresponding right eigenvectors

$$
r_{0}=\left(\begin{array}{c}
-\rho^{\gamma+1} \\
\rho^{\gamma} u \\
\kappa \gamma \rho^{\gamma}-u
\end{array}\right), \quad r_{1}=\left(\begin{array}{c}
1 \\
-\kappa \gamma \rho^{\gamma-1} \\
0
\end{array}\right), \quad r_{2}=\left(\begin{array}{l}
1 \\
0 \\
0
\end{array}\right) \text {. }
$$

The first family and the third family are linearly degenerate, while the second family is genuinely nonlinear.

Moreover, on the hypersurface, which is called the resonance hypersurface,

$$
C_{+}^{\kappa}:=\left\{(\rho, u, \kappa) \mid u=\kappa \gamma \rho^{\gamma}\right\}
$$

the second and the first characteristic speeds coincide, i.e.,

$$
\lambda_{1}(U)=\lambda_{0}(U)=0
$$


and

$$
\lambda_{2}(U)=\lambda_{0}(U)=0,
$$

on the hypersurface

$$
C_{-}:=\{(\rho, u, \kappa) \mid u=0\} .
$$

Now, we use curve (2.4) to separate the phase space $\{\rho \geq 0, u \geq 0\}$ into two parts $G_{1}^{\kappa}$ and $G_{2}^{\kappa}$, where

$$
\left\{\begin{array}{l}
G_{1}^{\kappa}:=\left\{U \mid \lambda_{1}(U)>0\right\}=\left\{U \mid u>\kappa \gamma \rho^{\gamma}\right\}, \\
G_{2}^{\kappa}:=\left\{U \mid \lambda_{1}(U)<0 \leq \lambda_{2}(U)\right\}=\left\{U \mid 0 \leq u<\kappa \gamma \rho^{\gamma}\right\} .
\end{array}\right.
$$

In order to analyze the solutions to system (1.4), we need to look at the wave curves. Since equations (1.4) and the Riemann data are invariant under uniform stretching of coordinates

$$
(x, t) \rightarrow(\alpha x, \alpha t), \quad \alpha>0 \text { is constant. }
$$

By taking the self-similar transform $\xi=x / t$, Riemann problem (1.4) and (1.5) is changed into the boundary value problem of the ordinary differential equation:

$$
\left\{\begin{array}{l}
-\xi \rho_{\xi}+(\rho u)_{\xi}=0 \\
-\xi(\rho(u+p))_{\xi}+(\rho u(u+p))_{\xi}=0 \\
-\xi \kappa_{\xi}=0
\end{array}\right.
$$

with $(\rho, u, \kappa)(\infty)=\left(\rho_{R}, u_{R}, \kappa_{R}\right)$ and $(\rho, u, \kappa)(-\infty)=\left(\rho_{L}, u_{L}, \kappa_{L}\right)$.

For smooth solutions, equations (2.7) can be rewritten as

$$
\left(\begin{array}{ccc}
u-\xi & \rho & 0 \\
(u-\xi) p_{\rho} & u-\xi & u p_{\kappa} \\
0 & 0 & -\xi
\end{array}\right)\left(\begin{array}{l}
\rho \\
u \\
\kappa
\end{array}\right)_{\xi}=0
$$

It follows from (2.8) that besides the constant solution $(\rho>0)$, it provides a rarefaction wave which is a continuous solution of (2.8) in the form $U(\xi)$. Given a left state $U_{0}=$ $\left(\rho_{0}, u_{0}, \kappa_{0}\right)$, the rarefaction wave curve is the sets of all right states $U=(\rho, u, \kappa)$ that can be connected to the left by a rarefaction wave in the second-family, which are as follows:

$$
R\left(U_{0}\right):\left\{\begin{array}{l}
\xi=\lambda_{1}=u-\kappa \gamma \rho^{\gamma}, \quad \rho<\rho_{0}, \\
u+\kappa \rho^{\gamma}=u_{0}+\kappa_{0} \rho_{0}^{\gamma} \Longleftrightarrow u+p=u_{0}+p_{0} \\
\kappa=\kappa_{0},
\end{array}\right.
$$

where $p_{0}=\kappa_{0} \rho_{0}^{\gamma}$ and $p=\kappa \rho^{\gamma}$. 
For a bounded discontinuous solutions, the Rankine-Hugoniot conditions are

$$
\left\{\begin{array}{l}
-\sigma[\rho]+[\rho u]=0, \\
-\sigma[\rho(u+p)]+[\rho u(u+p)]=0 \\
-\sigma[\kappa]=0
\end{array}\right.
$$

where and in what follows we use the notation $[h]=h_{+}-h_{-}$with $h_{-}=h(x(t)-0, t)$ and $h_{+}=h(x(t)+0, t)$, and $\sigma=\frac{d x}{d t}$ is the velocity of the discontinuity. We obtain from (2.10) that

$$
\sigma=0 \quad \text { or } \quad[\kappa]=0 .
$$

When $[\kappa]=0$, by direct calculation and the Lax shock inequalities, the possible states $U=(\rho, u, \kappa)$ that can be connected to the left state $U_{0}$ on a shock wave are given by

$$
S\left(U_{0}\right): \begin{cases}\sigma=u-\frac{\rho_{0}\left(\kappa \rho^{\gamma}-\kappa_{0} \rho_{0}^{\gamma}\right)}{\rho-\rho_{0}}, & \rho>\rho_{0}, \\ u+\kappa \rho^{\gamma}=u_{0}+\kappa_{0} \rho_{0}^{\gamma} & \Longleftrightarrow u+p=u_{0}+p_{0}, \\ \kappa=\kappa_{0} . & \end{cases}
$$

If $\kappa=$ constant, then system (1.4) belongs to the Temple class, for which the rarefaction wave curves coincide with the shock curves in the phase plane [35, 36].

The third characteristic family is linearly degenerate, that is, $[\kappa]=0$ and $\sigma=u$. The corresponding curve is called contact discontinuity, denoted $J$. The curve, which is the set of states $U=(\rho, u, \kappa)$ that can be connected to $U_{0}$ by the contact discontinuity, is

$$
J\left(U_{0}\right):\left\{\begin{array}{l}
u=u_{0} \\
\kappa=\kappa_{0} .
\end{array}\right.
$$

Remark 1 For a special shock, i.e., the velocity of shock $\sigma=0$, we call it stationary shock wave, denoted by $S_{0}$.

To facilitate our construction, for a given state $U_{0}=\left(\rho_{0}, u_{0}, \kappa_{0}\right)$, we define the wave curves $\mathcal{W}_{i}(i=1,2)$ as follows:

$$
\begin{aligned}
& \mathcal{W}_{1}\left(U_{0}\right): \quad u=\omega_{1}\left(U_{0}, \rho\right)= \begin{cases}u_{0}+\kappa_{0}\left(\rho^{\gamma}-\rho_{0}^{\gamma}\right), & \rho \leq \rho_{0}, \\
u_{0}+\kappa_{0}\left(\rho^{\gamma}-\rho_{0}^{\gamma}\right), & \rho>\rho_{0},\end{cases} \\
& \mathcal{W}_{2}\left(U_{0}\right): \quad u=\omega_{2}\left(U_{0}, \rho\right)=u_{0} .
\end{aligned}
$$

When $\sigma=0$ and $[\kappa] \neq 0$, we call the curve stationary contact wave, denoted by $\mathcal{W}_{0}$. For a given left state $U_{0}$ and some $\kappa$, the sets, which can be connected to $U_{0}$ by $\mathcal{W}_{0}$, are

$$
\mathcal{W}_{0}\left(U_{0}, \kappa\right):\left\{\begin{array}{l}
\rho u=\rho_{0} u_{0} \\
u+p=u_{0}+p_{0}
\end{array}\right.
$$

where $p=\kappa \rho^{\gamma}$ and $p_{0}=\kappa_{0} \rho_{0}^{\gamma}$. 


\section{The properties of wave curves}

To solve (1.4)-(1.5), we project all the wave curves on the $(\rho, u)$-plane. Now, let us investigate properties of the stationary shock wave and stationary contact wave along the lines of [27-29] with some novel information. First, we give the following lemma.

Lemma 3.1 For any $U_{L}\left(\rho_{L}, u_{L}, \kappa\right) \in G_{1}^{\kappa}$, we have:

(1) The speed of shock wave $\sigma\left(U_{L}, U\right)$ is decreasing with respect to $\rho$, where $U=(\rho, u, \kappa) \in S\left(U_{L}\right)$. Moreover, there exists a unique state $U_{S} \in S\left(U_{L}\right) \cap G_{2}^{\kappa}$ such that $\sigma\left(U_{L}, U_{S}\right)=0$, where the state $U_{S}$ satisfies

$$
\left\{\begin{array}{l}
u_{S}=u_{L}+\kappa\left(\rho_{L}^{\gamma}-\rho_{S}^{\gamma}\right), \\
u_{S}=\frac{\rho_{L} u_{L}}{\rho_{S}} .
\end{array}\right.
$$

(2) $\rho_{S}$ is a decreasing function with respect to $\rho_{L}$ along the shock.

Proof For statement (1), we use (2.12) to get $\frac{d u}{d \rho}=-\kappa \gamma \rho^{\gamma-1}$ and

$$
\sigma\left(U_{L}, U\right)=u-\frac{\kappa \rho_{L}\left(\rho^{\gamma}-\rho_{L}^{\gamma}\right)}{\rho-\rho_{L}} .
$$

Differentiating both sides of (3.2) with respect to $\rho$ gives

$$
\begin{aligned}
\frac{d \sigma}{d \rho} & =\frac{d u}{d \rho}-\frac{\kappa \rho_{L}}{\left(\rho-\rho_{L}\right)^{2}}\left((\gamma-1) \rho^{\gamma}-\gamma \rho_{L} \rho^{\gamma-1}+\rho_{L}^{\gamma}\right) \\
& =-\frac{\kappa}{\left(\rho-\rho_{L}\right)^{2}}\left(\gamma \rho^{\gamma+1}-(\gamma+1) \rho_{L} \rho^{\gamma}+\rho_{L}^{\gamma+1}\right) .
\end{aligned}
$$

Setting $f(\rho)=\gamma \rho^{\gamma+1}-(\gamma+1) \rho_{L} \rho^{\gamma}+\rho_{L}^{\gamma+1}$, we have $f^{\prime}(\rho)=\gamma(\gamma+1) \rho^{\gamma-1}\left(\rho-\rho_{L}\right)>0$ for $\rho>\rho_{L}$. Together with the fact that $f\left(\rho_{L}\right)=0$, one can obtain

$$
f(\rho)>0 \text { for } \rho>\rho_{L} .
$$

Combining (3.3) and (3.4) gives that the speed of shock $\sigma\left(U_{L}, U\right)$ is decreasing with respect to $\rho$.

From (3.2) and $U_{L} \in G_{1}^{\kappa}$, we have

$$
\begin{aligned}
\lim _{\rho \rightarrow \rho_{L}} \sigma\left(U_{L}, U\right) & =\lim _{\rho \rightarrow \rho_{L}}\left(u-\frac{\rho_{L}\left(p(\rho, \gamma)-p\left(\rho_{L}, \gamma\right)\right)}{\rho-\rho_{L}}\right) \\
& =u_{L}-\kappa \gamma \rho_{L}^{\gamma}>0 .
\end{aligned}
$$

It is easy to see that $\sigma\left(U_{L}, U\right)<0$ for $U=(\rho, 0, \kappa) \in S\left(U_{L}\right)$. Based on these properties of the function $\sigma\left(U_{L}, U\right)$, there exists a unique state $U_{S} \in S\left(U_{L}\right) \cap G_{2}^{\kappa}$ such that the speed of shock wave $\sigma\left(U_{L}, U_{S}\right)=0$, which yields statement (1).

We now show statement (2). Denote $\omega_{L}=u_{L}+p_{L}$. By statement (1), we have $U_{L} \in G_{1}^{K}$ and $U_{S} \in G_{2}^{\kappa}$, which imply that $u_{L}>\kappa \gamma \rho_{L}^{\gamma}$ and $u_{S}<\kappa \gamma \rho_{S}^{\gamma}$. We obtain from (3.1) that $\omega_{S}=$ $\omega_{L}=u_{S}+p_{S}$ and

$$
\rho_{L}\left(\omega_{L}-\kappa \rho_{L}^{\gamma}\right)=\rho_{S}\left(\omega_{L}-\kappa \rho_{S}^{\gamma}\right) .
$$


Differentiating both sides of the above equality with respect to $\rho_{L}$ gives

$$
u_{L}-\kappa \gamma \rho_{L}^{\gamma}=\left(u_{S}-\kappa \gamma \rho_{S}^{\gamma}\right) \frac{d \rho_{S}}{d \rho_{L}}
$$

which indicates $\frac{d \rho_{S}}{d \rho_{L}}<0$. We achieve statement (2), and the lemma is completely proved.

Next, we discuss the properties of the stationary contact wave.

Given a state $U_{0}=\left(\rho_{0}, u_{0}, \kappa_{0}\right)$, assume $U=(\rho, u, \kappa)$ to be the corresponding right-hand state of the stationary contact wave issuing from the left state $U_{0}$. We obtain from (2.16) that

$$
\left\{\begin{array}{l}
\rho u=\rho_{0} u_{0}, \\
u+\kappa \rho^{\gamma}=u_{0}+\kappa_{0} \rho_{0}^{\gamma},
\end{array}\right.
$$

which gives

$$
\varphi(\rho):=\kappa \rho^{\gamma+1}-\omega_{0} \rho+m_{0}=0,
$$

where $\omega_{0}=u_{0}+\kappa_{0} \rho_{0}^{\gamma} \geq 0$ and $m_{0}=\rho_{0} u_{0} \geq 0$. It follows from (3.6) that $\varphi^{\prime}(\rho)=\kappa(\gamma+1) \rho^{\gamma}-$ $\omega_{0}$. So $\varphi^{\prime}(\rho)=0$ implies $\kappa(\gamma+1) \rho^{\gamma}=\omega_{0}$, which gives

$$
\rho_{*}:=\rho_{*}\left(U_{0}, \kappa\right)=\left(\frac{\omega_{0}}{\kappa(\gamma+1)}\right)^{\frac{1}{\gamma}}>0 .
$$

Then $\varphi^{\prime}(\rho)<0$ for $0<\rho<\rho_{*}$ and $\varphi^{\prime}(\rho)>0$ for $\rho>\rho_{*}$. Therefore

$$
\begin{aligned}
\min _{\rho>0} \varphi(\rho) & =\varphi\left(\rho_{*}\right)=\rho_{*}\left(\kappa \rho_{*}^{\gamma}-\omega_{0}\right)+m_{0} \\
& =-\kappa \gamma \rho_{*}^{\gamma+1}+m_{0} .
\end{aligned}
$$

It is easy to see that $\varphi\left(\rho_{*}\right)<0$ is equivalent to

$$
\rho_{*} \geq\left(\frac{m_{0}}{\kappa \gamma}\right)^{\frac{1}{\gamma+1}}=: \rho_{\min }\left(U_{0}, \kappa\right)=\rho_{\min }
$$

which implies

$$
\kappa \leq \frac{\omega_{0}}{\gamma+1}\left(\frac{\omega_{0} \gamma}{m_{0}(\gamma+1)}\right)^{\gamma}=: \kappa_{\max }\left(U_{0}\right)=\kappa_{\max }
$$

Obviously, we have $\varphi(0)=m_{0}>0$. In addition, we can take $\bar{\rho}>\left(\frac{\omega_{0}}{\kappa}\right)^{\frac{1}{\gamma}}$ such that $\varphi(\bar{\rho})>0$.

Based on these observations, we arrive at the following lemma.

Lemma 3.2 Given a state $U_{0}=\left(\rho_{0}, u_{0}, \kappa_{0}\right)$ and some $\kappa$, then a stationary contact wave $\mathcal{W}_{0}\left(U_{L}, \kappa\right)$ issuing from $U_{0}$ to some $U=(\rho, u, \kappa)$ exists if and only if equation (3.6) admits a root, which is equivalent to that condition (3.10) holds. Moreover, when $\kappa<\kappa_{\max }$, equation (3.6) has two roots $\rho_{1}(\kappa)$ and $\rho_{2}(\kappa)$ such that $\rho_{1}(\kappa)<\rho_{*}<\rho_{2}(\kappa)$.

We continue to consider the properties of the stationary contact wave. 
Lemma 3.3 Let $U_{0}$ and $U$ be described as in Lemma 3.2. We have the following results:

(1) $\kappa_{\max } \geq \kappa_{0}, \kappa_{\max }=\kappa_{0}$ if and only if $U_{0} \in C_{+}^{\kappa_{0}}$.

(2) If $\kappa \leq \kappa_{\max }$ and $u_{0}>0$, then $\rho_{1}(\kappa) \leq \rho_{*} \leq \rho_{2}(\kappa)$, where $\rho_{1}(\kappa)$ and $\rho_{2}(\kappa)$ are two roots of (3.6).

(3) Let $\kappa \leq \kappa_{\max }, u_{0}>0$ and $U_{i}(\kappa)=\left(\rho_{i}(\kappa), u_{i}(\kappa), \kappa\right)$, where $u_{i}(\kappa)=\rho_{0} u_{0} / \rho_{i}(\kappa), i=1,2$, $\rho_{1}(\kappa)$ and $\rho_{2}(\kappa)$ are described as the statement in (2), then

$$
U_{1}(\kappa) \in G_{1}^{\kappa} \quad \text { and } \quad U_{2}(\kappa) \in G_{2}^{\kappa}
$$

Proof To prove (1), we assume $u_{0}=\alpha \kappa_{0} \gamma \rho_{0}^{\gamma}, \alpha>0$, then $\omega_{0}=u_{0}+\kappa_{0} \rho_{0}^{\gamma}=(\alpha \gamma+1) \kappa_{0} \rho_{0}^{\gamma}$ and $m_{0}=\rho_{0} u_{0}=\alpha \gamma \kappa_{0} \rho_{0}^{\gamma+1}$. Substituting these into (3.10) gives

$$
\kappa_{\max }=\kappa_{0} \frac{1}{\alpha^{\gamma}}\left(\frac{1+\alpha \gamma}{\gamma+1}\right)^{\gamma+1} .
$$

When $\alpha=1$, that is, $U_{0} \in C_{+}^{\kappa_{0}}$, then $\kappa_{\max }=\kappa_{0}$. When $\alpha \neq 1$, we introduce the function

$$
f(\alpha)=\frac{1}{\alpha^{\gamma}}\left(\frac{1+\alpha \gamma}{\gamma+1}\right)^{\gamma+1}>0
$$

and achieve

$$
(\ln f(\alpha))^{\prime}=\frac{f^{\prime}(\alpha)}{f(\alpha)}=\frac{(\alpha-1) \gamma}{a(1+\alpha \gamma)} .
$$

Obviously, if $\alpha>1, f^{\prime}(\alpha)>0$, which shows $f(\alpha)>f(1)=1$. If $0<\alpha<1, f^{\prime}(\alpha)<0$, which implies $f(\alpha)>f(1)=1$. So $f(\alpha)>f(1)=1$ for $\alpha \neq 1$, which shows $\kappa_{\max }>\kappa_{0}$. Therefore, we demonstrate statement (1).

Assertion (2) can be easily deduced from the above argument.

Now, we check assertion (3). Let us define the function

$$
\psi(\rho)=u-\kappa \gamma \rho^{\gamma} .
$$

Together with (3.9), we obtain from (3.13) that $\psi\left(\rho_{\min }\right)=0$, which implies $\left(\rho_{\min }, u_{\min }, \kappa\right) \in$ $C_{+}^{\kappa}$, where $u_{\min }=m_{0} / \rho_{\min }$. Moreover, we have $\psi^{\prime}(\rho)=-\frac{m_{0}}{\rho^{2}}-\kappa \gamma^{2} \rho^{\gamma-1}<0$. So, in order to demonstrate $U_{1}(\kappa) \in G_{1}^{\kappa}$ and $U_{2}(\kappa) \in G_{2}^{\kappa}$, it is sufficient to prove

$$
\psi\left(\rho_{1}(\kappa)\right)>0 \quad \text { and } \quad \psi\left(\rho_{2}(\kappa)\right)<0
$$

which is equivalent to

$$
\rho_{1}(\kappa)<\rho_{\min }<\rho_{2}(\kappa) .
$$

From (3.6), we have

$$
\varphi\left(\rho_{\min }\right)=\frac{(\gamma+1) m_{0}}{\gamma}-\omega_{0} \rho_{\min } .
$$

Then, a straightforward computation shows that the condition $\kappa<\kappa_{\max }\left(U_{0}\right)$ is equivalent to $\varphi\left(\rho_{\min }\right)<0$. This together with the fact that $\varphi(\rho)>0$ for $\rho<\rho_{1}(\kappa)$ or $\rho>\rho_{2}(\kappa)$, 
and $\varphi(\rho)<0$ for $\rho_{1}(\kappa)<\rho<\rho_{2}(\kappa)$, establishes (3.15). This completes the proof of the lemma.

We next establish a lemma that plays a key role in construction of the Riemann solution.

Lemma 3.4 Let $U_{0}$ and $U$ be described as in Lemma 3.2, $\rho_{1}(\kappa)$ and $\rho_{2}(\kappa)$ be two roots of (3.6).

(1) If $\kappa<\kappa_{0}$ and $u_{0}>0$, then $\rho_{1}(\kappa)<\rho_{0}<\rho_{2}(\kappa)$. Moreover, when $U_{0} \in G_{2}^{\kappa_{0}}$, we have $U_{2}=\left(\rho_{2}(\kappa), u_{2}(\kappa), \kappa\right) \in G_{2}^{\kappa}$, where $u_{2}(\kappa)=m_{0} / \rho_{2}(\kappa)$.

(2) If $\kappa_{0}<\kappa<\kappa_{\max }$ and $u_{0}>0$, then

$$
\rho_{0}<\rho_{1}(\kappa) \quad \text { for } U_{0} \in G_{1}^{\kappa} ; \quad \rho_{0}>\rho_{2}(\kappa) \quad \text { for } U_{0} \in G_{2}^{\kappa} \text {. }
$$

Proof If $\kappa<\kappa_{0}$, then $\kappa<\kappa_{0} \leq \kappa_{\max }\left(U_{0}\right)$ and $\varphi\left(\rho_{0}\right)=\left(\kappa-\kappa_{0}\right) \rho_{0}^{\gamma+1}<0$, which implies $\rho_{1}(\kappa)<$ $\rho_{0}<\rho_{2}(\kappa)$.

It follows from (3.9) and (3.13) that

$$
\psi\left(\rho_{\min }\right)=\frac{1}{\rho_{\min }}\left(m_{0}-\kappa \gamma \rho_{\min }^{\gamma+1}\right)=0 .
$$

Since $\varphi\left(\rho_{2}\right)=0$, that is, $\kappa \rho_{2}^{\gamma+1}-\omega_{0} \rho_{2}+m_{0}=0$, we obtain

$$
\kappa \rho_{2}^{\gamma}=-\frac{m_{0}}{\rho_{2}}+\omega_{0} .
$$

Recalling the definition of the function $\psi(\rho)$ and $u_{2}=m_{0} / \rho_{2}<u_{0}$ for $\rho_{2}>\rho_{0}$, we observe

$$
\begin{aligned}
\psi\left(\rho_{2}\right) & =u_{2}-\kappa_{0} \gamma \rho_{0}^{\gamma}+\gamma\left(\frac{m_{0}}{\rho_{2}}-u_{0}\right) \\
& <u_{0}-\kappa_{0} \gamma \rho_{0}^{\gamma}<0
\end{aligned}
$$

for $U_{0} \in G_{2}^{\kappa_{0}}$. Furthermore, a simple calculation shows

$$
\frac{d \psi}{d \rho}=\frac{d u}{d \rho}-\kappa \gamma^{2} \rho^{\gamma-1}<0 .
$$

Combining (3.16) with (3.18)-(3.19) shows $\rho_{2}>\rho_{\min }$, which implies $U_{2} \in G_{2}^{\kappa}$.

Next, we prove assertion (2). It is derived from (3.9) and (3.13) that

$$
\psi\left(\rho_{0}\right)=u_{0}-\kappa \gamma \rho_{0}^{\gamma}>0
$$

for $U_{0} \in G_{1}^{\kappa}$ and

$$
\psi\left(\rho_{\min }\right)=u-\kappa \gamma \rho_{\min }^{\gamma}=0
$$

where $u=m_{0} / \rho_{\min }$. In addition, we have

$$
\psi^{\prime}(\rho)=-\frac{m_{0}}{\rho^{2}}-\kappa \gamma^{2} \rho^{\gamma-1}<0 .
$$

This together with (3.20) and (3.21) shows $\rho_{0}<\rho_{\min }$. 
By the argument of Lemma 3.3, we know that $\varphi\left(\rho_{\min }\right)<0$ is equivalent to $\kappa<\kappa_{\max }$. Moreover, since $\varphi\left(\rho_{0}\right)=\left(\kappa-\kappa_{0}\right) \rho_{0}^{\gamma+1}>0$, which together with the above observation gives $\rho_{0}<\rho_{1}(\kappa)$ for $U_{0} \in G_{1}^{\kappa}$.

If $U_{0} \in G_{2}^{\kappa}$, then

$$
\psi\left(\rho_{0}\right)=u_{0}-\kappa \gamma \rho_{0}^{\gamma}<0, \quad \psi\left(\rho_{\min }\right)=0, \quad \psi^{\prime}(\rho)<0
$$

which implies

$\rho_{0}>\rho_{\min }$

Furthermore, we easily obtain $\varphi\left(\rho_{0}\right)=\left(\kappa-\kappa_{0}\right) \rho_{0}^{\gamma+1}>0$ and $\varphi\left(\rho_{\min }\right)<0$. Combining this and (3.22) achieves $\rho_{0}>\rho_{2}(\kappa)$. So the second assertion of the lemma is true.

Remark 2 Lemma 3.4 answers the first problem as mentioned in Sect. 1.

\section{The TV-condition}

Based on the assertions in Lemmas 3.2 and 3.3, there are two possible stationary contact waves issuing from a given state $U_{0}$ to a state with another entropy value $\kappa$. Thus, we need to impose an admissibility condition to select a unique solution.

In order to use TV-condition (1.6), we need the following two lemmas.

Lemma 4.1 For $U_{L} \in C_{+}^{\kappa_{L}}$ and $\kappa_{L}>\kappa_{R}$, denote $H_{L}: \rho u=\rho_{L} u_{L}, C_{L}: u=\omega_{L}-\kappa_{L} \rho^{\gamma}$, and $C_{R}: u=\omega_{L}-\kappa_{R} \rho^{\gamma}$, and suppose that the curve $H_{L}$ intersects the curve $C_{R}$ at two states $U_{\alpha}$, $U_{\beta}$ and $\rho_{\alpha}<\rho_{\beta}$ (see Fig. 1), then

(1) the curve $H_{L}$ is tangent to the curve $C_{L}$ at $U_{L}$,

(2) $\rho_{L}-\rho_{\alpha}<\rho_{\beta}-\rho_{L}$

Proof Since $U_{L} \in C_{+}^{\kappa_{L}}$, we have $u_{L}=\kappa_{L} \gamma \rho_{L}^{\gamma}$, and so $m_{L}=\rho_{L} u_{L}=\kappa_{L} \gamma \rho_{L}^{\gamma+1}$ and $\omega_{L}=u_{L}+p_{L}=$ $\kappa_{L}(\gamma+1) \rho_{L}^{\gamma}$.

Statement (1) is trivial. We need only to prove statement (2).

If $\kappa_{L}>\kappa_{R}$, then $\xi=\kappa_{L} / \kappa_{R}>1$. Setting $\rho=\theta \rho_{L}$, we obtain from (3.6) that

$$
\varphi(\rho)=\kappa_{R} \rho_{L}^{\gamma+1}\left(\theta^{\gamma+1}-\xi(\gamma+1) \theta+\xi \gamma\right)=0
$$

Figure $1 U_{L} \in C_{+}^{\kappa_{L}}$ and $\kappa_{L}>\kappa_{R}$

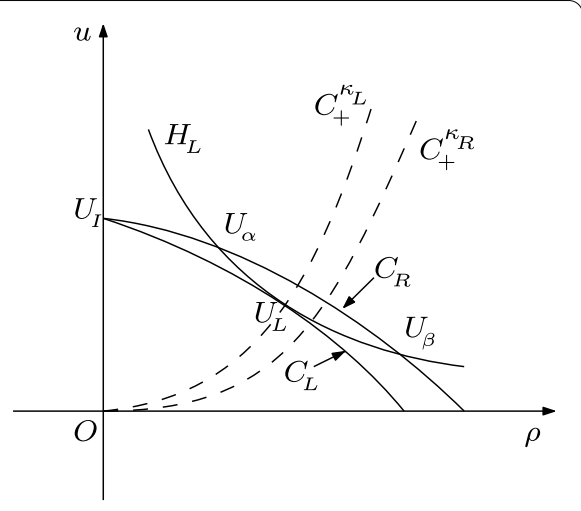


Figure 2 The graphs of $f_{1}(\theta), f_{2}(\theta)$, and $f_{3}(\theta)$

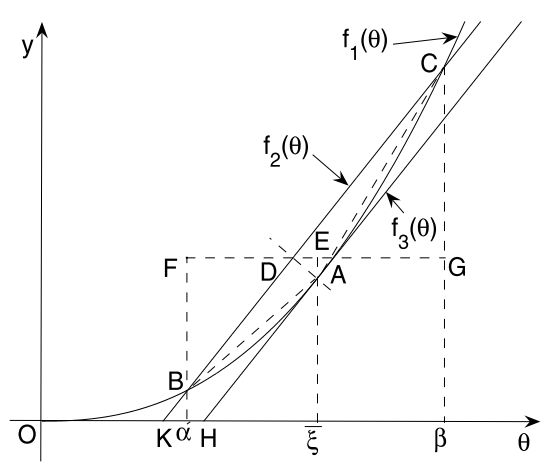

Let

$$
f(\theta)=\theta^{\gamma+1}-\xi(\gamma+1) \theta+\xi \gamma=0,
$$

and rearrange (4.1) to get

$$
\theta^{\gamma+1}=\xi(\gamma+1)\left(\theta-\frac{\gamma}{\gamma+1}\right)
$$

We easily see that equation (4.2) has two roots, denoted by $\alpha$ and $\beta$. They satisfy $0<\alpha<1$ and $\beta>\xi^{\frac{1}{\gamma}}>1$. So statement (2) is equivalent to $1-\alpha<\beta-1$, that is, $\alpha+\beta>2$.

Denote $f_{1}(\theta)=\theta^{\gamma+1}$ and $f_{2}(\theta)=\xi(\gamma+1)\left(\theta-\frac{\gamma}{\gamma+1}\right)$. The graphs of $y=f_{1}(\theta)$ and $y=f_{2}(\theta)$ are shown in Fig. 2. The $y=f_{2}(\theta)$ intersects the $y=f_{1}(\theta)$ at points $B$ and $C$, where $\theta_{B}=\alpha$ and $\theta_{C}=\beta$. The straight line $A H$ is $y=f_{3}(\theta)=\xi(\gamma+1)\left(\theta-\xi^{1 / \gamma}\right)+\xi^{(\gamma+1) / \gamma}$, which is a tangent line of $y=f_{1}(\theta)$ at point $A=A\left(\xi^{1 / \gamma}, \xi^{(\gamma+1) / \gamma}\right)$. Assume that $y=f_{2}(\theta)$ and $y=f_{3}(\theta)$ intersect the $\theta$-axis at points $H$ and $K$ respectively. Obviously, at two points $H$ and $K$, we have $\theta_{H}=\frac{\gamma}{\gamma+1} \xi^{1 / \gamma}$ and $\theta_{K}=\frac{\gamma}{\gamma+1}$, see Fig. 2 .

In Fig. 2, both the straight lines $B F$ and $C G$ are perpendicular to the $\theta$-axis and $F G$ is parallel to the $\theta$-axis, which intersects the $B C$ at point $D$. The $A E$ is perpendicular to the $F G$ at point $E$. By the property of the function $y=f_{1}(\theta)$ and the fact that $A H$ is parallel to $B C$, we have that the length of the line segment $C D$ is greater than that of the line segment $B D$, which implies

$$
\theta_{G}-\theta_{D}>\theta_{D}-\theta_{F}
$$

From Fig. 2, we obtain

$$
\left\{\begin{array}{l}
\beta=\theta_{C}=\theta_{G}=\theta_{A}+\left(\theta_{G}-\theta_{D}\right)-\left(\theta_{E}-\theta_{D}\right) \\
\alpha=\theta_{B}=\theta_{F}=\theta_{A}-\left(\theta_{D}-\theta_{F}\right)-\left(\theta_{E}-\theta_{D}\right) \\
\theta_{H}-\theta_{K}>\theta_{E}-\theta_{D}
\end{array}\right.
$$

Combining (4.3) and (4.4) gives

$$
\alpha+\beta>2 \xi^{\frac{1}{\gamma}}-2\left(\theta_{H}-\theta_{K}\right)=2+2\left(\xi^{\frac{1}{\gamma}}-1\right)-2 \frac{\gamma}{\gamma+1}\left(\xi^{\frac{1}{\gamma}}-1\right)>2
$$

for $\xi>1$ and $1<\gamma<2$. Then the lemma is verified. 


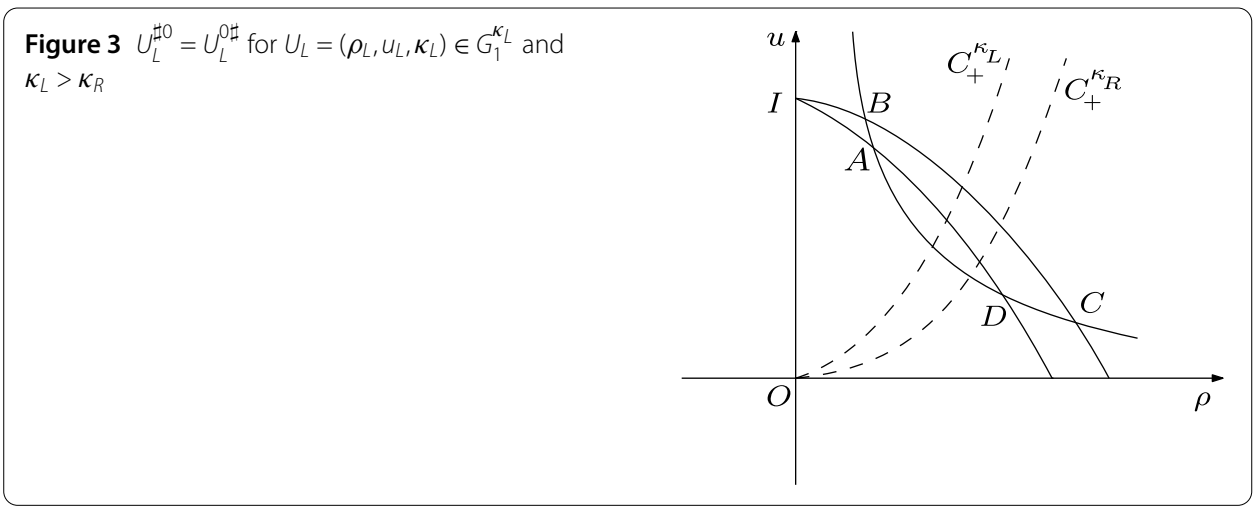

Before we get interesting results by using the following lemma, we first introduce some notations as follows.

\section{Notations}

(i) $W_{k}\left(U_{i}, U_{j}\right)$ denotes the $k$ th-wave connecting the left-hand state $U_{i}$ to the right-hand state $U_{j}, k=0,1,2$.

(ii) $U^{\sharp}$ denotes the state resulted by a zero-speed shock wave from $U$.

(iii) $U^{0}$ denotes the state resulted by a stationary contact wave from $U$.

Setting $\omega_{L}=u_{L}+p_{L}=u_{L}+\kappa_{L} \rho_{L}^{\gamma}$, from the point $A=\left(\rho_{L}, u_{L}, \kappa_{L}\right)$ in $(\rho, u)$-plane, we draw a curve $H_{L}: \rho u=\rho_{L} u_{L}$ and another curve $C_{L}: u=\omega_{L}-\kappa_{L} \rho^{\gamma}$ which intersects the $u$-axis at point $I=I\left(U_{I}\right)=I\left(0, \omega_{L}\right)$. From the point $I$, we draw a curve $C_{R}: u=\omega_{L}-\kappa_{R} \rho^{\gamma}$. We assume that the curve $H_{L}$ intersects with the curves $C_{R}$ and $C_{L}$ at points $B, C$, and $D$ respectively, see Fig. 3.

We obtain from Lemma 3.1 and (2.16) that the points $B$ and $C$ are determined by

$$
\left\{\begin{array}{l}
\rho_{L} u_{L}=\rho u, \\
u_{L}+p_{L}=u+p,
\end{array}\right.
$$

which implies $B=B\left(\rho_{L}^{0}, u_{L}^{0}, \kappa_{r}\right)$ and $C=C\left(\rho_{L}^{0 \sharp}, u_{L}^{0 \sharp}, \kappa_{R}\right)$, where $p=\kappa_{R} \rho^{\gamma}$.

In addition, the point $D$ also satisfies (4.5), where $p=\kappa_{L} \rho^{\gamma}$. From Lemma 3.1, we obtain $D=D\left(\rho_{L}^{\sharp}, u_{L}^{\sharp}, \kappa_{L}\right)$. Thus, $U_{L}^{\sharp 0}=\left(\rho_{L}^{\sharp 0}, u_{L}^{\sharp 0}, \kappa_{R}\right)$ satisfies

$$
\left\{\begin{array}{l}
\rho_{L}^{\sharp} u_{L}^{\sharp}=\rho u, \\
u_{L}^{\sharp}+p_{L}^{\sharp}=u+p,
\end{array}\right.
$$

where $p=\kappa_{R} \rho^{\gamma}$. So, we obtain $\left(\rho_{L}^{\sharp 0}, u_{L}^{\sharp 0}, \kappa_{R}\right)=\left(\rho_{L}^{0 \sharp}, u_{L}^{0 \sharp}, \kappa_{R}\right)$.

Based on the above argument and Lemma 4.1, we present an important lemma as follows.

Lemma 4.2 For a given state $U_{L}=\left(\rho_{L}, u_{L}, \kappa_{l}\right) \in G_{1}^{\kappa_{L}}, U_{L}^{0}, U_{L}^{\sharp}, U_{L}^{\sharp 0}$, and $U_{L}^{\sharp 0}$ described as above, we denote $A=A\left(U_{L}\right), B=B\left(U_{L}^{0}\right), D=D\left(U_{L}^{\sharp}\right)$, as shown in Fig. 3. Then, we have:

(1) $U_{L}^{\sharp 0}=U_{L}^{0 \sharp}$.

(2) $\left|\rho_{L}-\rho_{L}^{0}\right| \leq\left|\rho_{L}^{0 \sharp}-\rho_{L}^{\sharp}\right|$. 
Remark 3 Statement (1) of Lemma 4.2 is different from the case that in general $U_{L}^{\sharp 0} \neq U_{L}^{0 \sharp}$ in $[28,29]$ and results in possibly infinitely many solutions for certain initial data, see the following two subcases (A5) and (B5).

\section{The Riemann solution to (1.4) and (1.5) with (1.2)}

In this section, we use our TV-condition (1.6) to construct the corresponding Riemann solution. Moreover, the TV-condition helps us select a unique one among the possibly infinitely many solutions for certain Riemann data.

Next, we consider the corresponding Riemann for cases $\kappa_{L}>\kappa_{R}$ and $\kappa_{L}<\kappa_{R}$ and construct the solutions case by case.

\subsection{The Riemann solution for the case $\kappa_{L}>\kappa_{R}$}

We divide this case into three subcases: (I): $U_{L} \in G_{1}^{\kappa_{L}}$, (II): $U_{L} \in G_{2}^{\kappa_{L}}$, and (III): $U_{L} \in C_{+}^{\kappa_{L}}$.

(I) $U_{L} \in G_{1}^{\kappa_{L}}$

There is a stationary contact wave from the state $U_{L}\left(\kappa_{L}\right)$ to some state $U_{L}^{0}\left(\kappa_{R}\right)$ using $\rho_{1}\left(U_{L}, \kappa_{R}\right)$. Based on Lemma 3.1, there exists a state $U_{L}^{0 \sharp} \in W_{1}\left(U_{L}^{0}\right)$ such that $\sigma\left(U_{L}^{0}, U_{L}^{0 \sharp}\right)=$ $0, \sigma\left(U_{L}^{0}, U\right)>0$ for $u_{L}^{0 \sharp}<u<u_{L}^{0}$ and $\sigma\left(U_{L}^{0}, U\right)<0$ for $0 \leq u<u_{L}^{0 \sharp}$, where $U=\left(\rho, u, \kappa_{R}\right) \in$ $W_{1}\left(U_{L}^{0}\right)$, see Fig. 4 . In addition, we assume that the curve $W_{1}\left(U_{L}^{0}\right)$ intersects the $u$-axis at point $I=I\left(U_{I}\right)$, where $U_{I}=\left(0, u_{I}\right)$ and $u_{I}=\omega_{L}=u_{L}+p_{L}$. For a given right state $U_{R}=$ $\left(\rho_{R}, u_{R}, \kappa_{R}\right)$, we can divide construction of the Riemann solution into five subcases (A1)(A5), where
(A1): $u_{R}>u_{I}$,
(A2): $u_{L}^{0} \leq u_{R} \leq u_{I}$,
(A3): $u_{L}^{0 \sharp}<u_{R}<u_{L}^{0}$,
(A4): $0 \leq u_{R}<u_{L}^{0 \sharp}$,
(A5): $u_{R}=u_{L}^{0 \sharp}$.

(A1) $u_{R}>u_{I}$. For this case, the Riemann solution can be described as

$$
U_{L}+W_{0}\left(U_{L}, U_{L}^{0}\right)+U_{L}^{0}+R\left(U_{L}^{0}, U_{I}\right)+U_{V}+W_{2}\left(U_{V}, U_{R}\right)+U_{R}
$$

where the state $U_{V}$ denotes the Vacuum state, i.e.,

$$
U_{V}(\xi)= \begin{cases}U_{I}, & \xi \leq u_{I} \\ (0, u(\xi)), & u_{I}<\xi<u_{R} \\ \left(0, u_{R}\right), & u_{R} \leq \xi\end{cases}
$$

where $\xi=\frac{x}{t}$. The Riemann solution at $(x, t)$ space is shown in Fig. 5 .

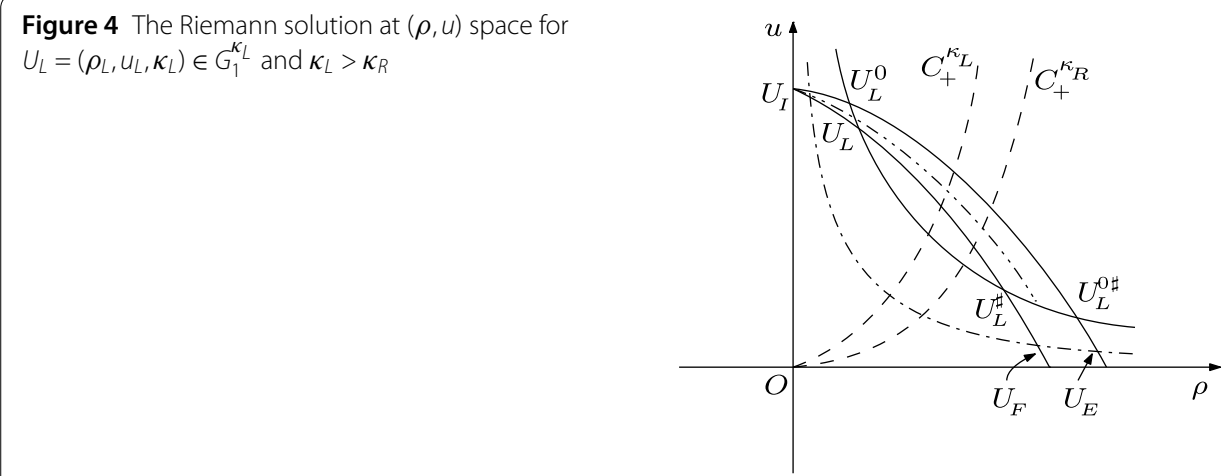



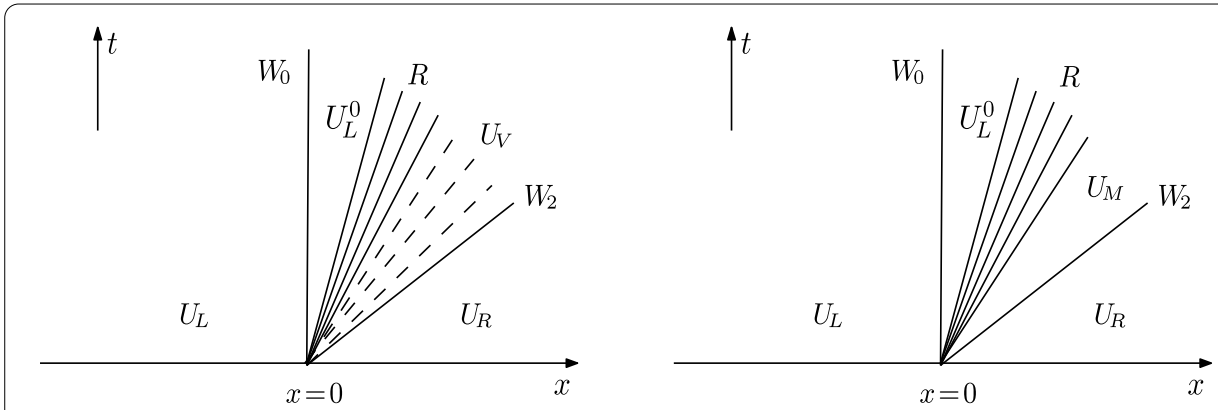

Figure 5 The Riemann solution at $(x, t)$ space for subcases (A1) (left) and (A2) (right), respectively
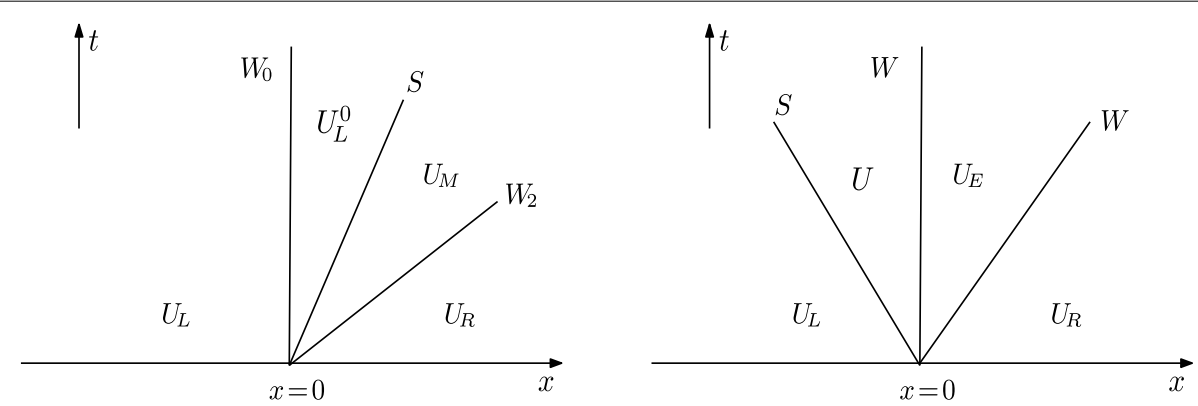

Figure 6 The Riemann solution at $(x, t)$ space for subcases (A3) (left) and (A4) (right), respectively

(A2) $u_{L}^{0} \leq u_{R} \leq u_{I}$. The Riemann solution can be expressed as

$$
U_{L}+W_{0}\left(U_{L}, U_{L}^{0}\right)+U_{L}^{0}+R\left(U_{L}^{0}, U_{M}\right)+U_{M}+W_{2}\left(U_{M}, U_{R}\right)+U_{R}
$$

where the state $U_{M}=\left(\rho_{M}, u_{M}, \kappa_{R}\right)$ satisfies

$$
\left\{\begin{array}{l}
u+\kappa_{R} \rho^{\gamma}=u_{L}^{0}+\kappa_{R}\left(\rho_{L}^{0}\right)^{\gamma}, \\
u=u_{R},
\end{array}\right.
$$

which is shown in Fig. 5.

(A3) $u_{L}^{0 \sharp}<u_{R}<u_{L}^{0}$. For this case, the Riemann solution can be described as

$$
U_{L}+W_{0}\left(U_{L}, U_{L}^{0}\right)+U_{L}^{0}+S\left(U_{L}^{0}, U_{M}\right)+U_{M}+W_{2}\left(U_{M}, U_{R}\right)+U_{R},
$$

where the state $U_{M}=\left(\rho_{M}, u_{M}, \kappa_{R}\right)$ satisfies (5.4), which is demonstrated in Fig. 6 .

(A4) $0 \leq u_{R}<u_{L}^{0 \sharp}$. In this subcase, by the following procedure, we construct the corresponding Riemann solution.

We draw a straight line $u=u_{R}$ from the right state $U_{R}$, which intersects the curve $C_{R}$ : $u=\omega_{L}-\kappa_{R} \rho^{\gamma}$ at point $E=E\left(U_{E}\right)$, where $\omega_{L}=u_{L}+p_{L}$ and $U_{E}=\left(\rho_{-}^{0}, u_{-}^{0}, \kappa_{R}\right)$ is given by

$$
\left\{\begin{array}{l}
u+\kappa_{R} \rho^{\gamma}=u_{L}+\kappa_{L} \rho_{L}^{\gamma}, \\
u=u_{R} .
\end{array}\right.
$$


And then, we draw a curve $H_{R}: \rho u=\rho_{-}^{0} u_{-}^{0}$ through the point $E$, which intersects the curve $C_{L}: u=\omega_{L}-\kappa_{L} \rho^{\gamma}$ at point $F=F\left(U_{F}\right)$, where $U_{F}=\left(\rho_{-}, u_{-}, \kappa_{L}\right)$ is determined by

$$
\left\{\begin{array}{l}
\rho u=\rho_{-}^{0} u_{-}^{0}, \\
u+\kappa_{L} \rho^{\gamma}=u_{-}^{0}+\kappa_{R}\left(\rho_{-}^{0}\right)^{\gamma}, \\
u<u_{L}^{\sharp} .
\end{array}\right.
$$

By Lemma 3.1, we have $\sigma\left(U_{L}, U_{F}\right)<0$. So, the Riemann solution can be described as

$$
U_{L}+S\left(U_{L}, U_{F}\right)+U_{F}+W_{0}\left(U_{F}, U_{E}\right)+U_{E}+W_{2}\left(U_{E}, U_{R}\right)+U_{R}
$$

see Fig. 6.

Remark 4 If we apply the monotonicity criterion introduced by LeFloch and Thanh in [27-29] to construct the Riemann solution for subcases (A1)-(A4), the corresponding results are consistent with the above results obtained by the TV-condition.

(A5) $u_{R}=u_{L}^{0 \sharp}$.

To begin with, from the point $I=I\left(U_{I}\right)$, we draw a curve $C_{\kappa}: u=\omega_{L}-\kappa \rho^{\gamma}$, which intersects the curve $H_{L}: \rho u=\rho_{L} u_{L}$ at points $M=M\left(U_{M}(\kappa)\right)$ and $N=N\left(U_{N}(\kappa)\right)$, where $\kappa_{R}<\kappa<\kappa_{L}$.

First, we use the monotonicity criterion to construct the Riemann solution. Then, the Riemann solution begins with a stationary contact wave from the state $U_{L}\left(\kappa_{L}\right)$ to some state $U_{M}(\kappa)$ using $\rho_{1}\left(U_{L}, \kappa\right)$. By Lemma 4.2 , we have $U_{N}=U_{M}^{\sharp}$; that is to say, there is a zero speed shock $S_{0}\left(U_{M}, U_{N}\right)$ from $U_{M}$ to $U_{N}$. And then, the $S_{0}$ is followed by a stationary contact wave $W_{0}\left(U_{N}, U_{L}^{0 \sharp}\right)$ from $U_{N}$ to $U_{L}^{0 \sharp}$. Here, the state $U_{L}^{0 \sharp}$ coincides in the following subcases (a), (b), and (c). Both $U_{M}$ and $U_{N}$ are not constant states and can be seen as the functions of the variable $\kappa$. Thereby, the corresponding Riemann solution, shown in Fig. 7, can be described as

$$
\begin{aligned}
(\mathrm{a}): U_{L} & +\underbrace{W_{0}\left(U_{L}, U_{M}\right)+U_{M}+S_{0}\left(U_{M}, U_{N}\right)+U_{N}+W_{0}\left(U_{N}, U_{L}^{0 \sharp}\right)}_{x=0} \\
& +U_{L}^{0 \sharp}+W_{2}\left(U_{L}^{0 \sharp}, U_{R}\right)+U_{R} .
\end{aligned}
$$

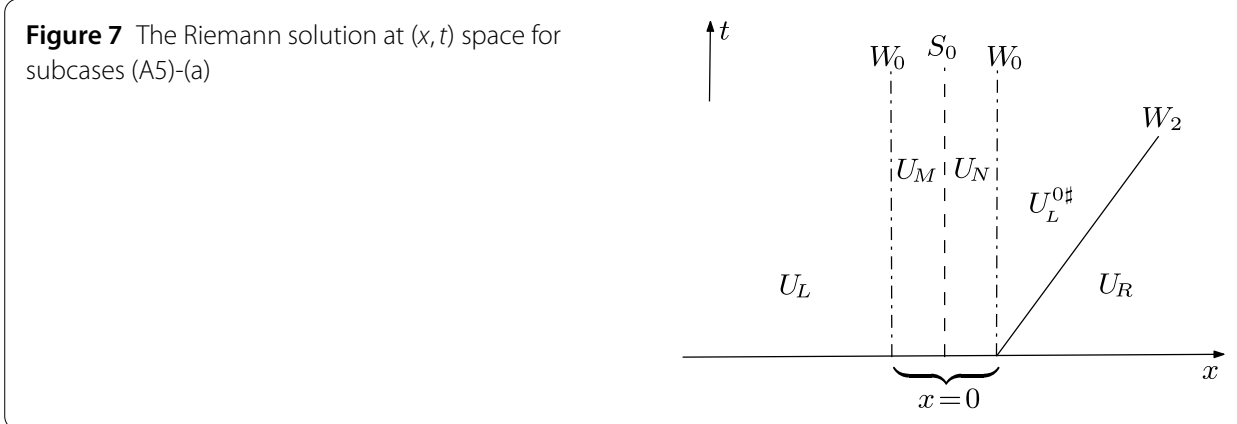




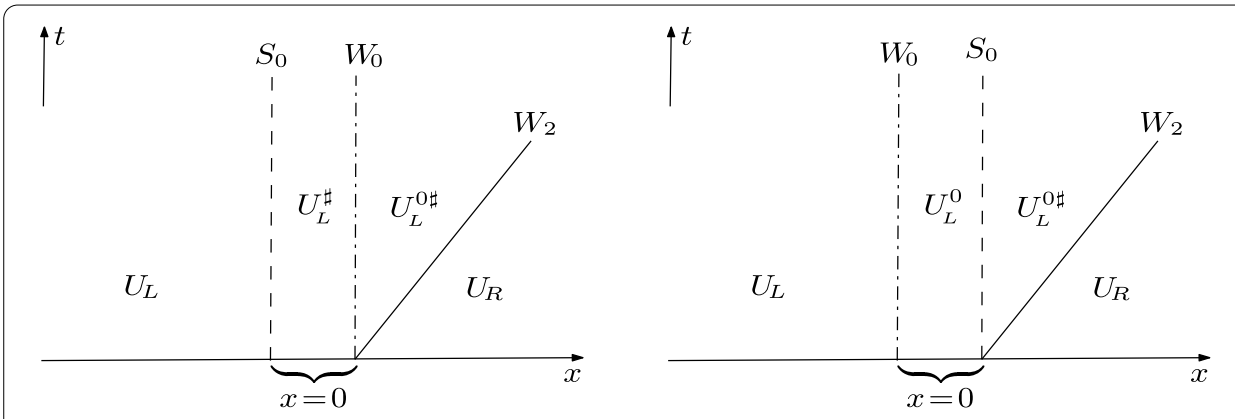

Figure 8 The Riemann solution at $(x, t)$ space for subcases (A5)-(b) (left) and (A5)-(c) (right), respectively

If $\kappa=\kappa_{L}$, together with Lemma 4.2, we can see from Fig. 8 that the Riemann solution can be expressed as

(b): $\quad U_{L}+\underbrace{S_{0}\left(U_{L}, U_{L}^{\sharp}\right)+U_{L}^{\sharp}+W_{0}\left(U_{L}^{\sharp}, U_{L}^{0 \sharp}\right)}_{x=0}+U_{L}^{0 \sharp}+W_{2}\left(U_{L}^{0 \sharp}, U_{R}\right)+U_{R}$.

If $\kappa=\kappa_{R}$, it is apparent from the previous discussion that the Riemann solution is

(c): $\quad U_{L}+\underbrace{W_{0}\left(U_{L}, U_{L}^{0}\right)+U_{L}^{0}+S_{0}\left(U_{L}^{0}, U_{L}^{0 \sharp}\right)}_{x=0}+U_{L}^{0 \sharp}+W_{2}\left(U_{L}^{0 \sharp}, U_{R}\right)+U_{R}$.

So, we see that the monotonicity criterion cannot select a unique one among infinitely many solutions.

Now, we use TV-condition (1.6) to consider subcase (A5). From Lemma 4.2, the minimum of $\operatorname{TV}_{W_{0}}(\rho)$ is $\left|\rho_{L}-\rho_{L}^{0}\right|$, which indicates that the Riemann solution is described as (5.9). Thus, under TV-condition (1.6), we obtain the uniqueness of the Riemann solution for this subcase.

(II) $U_{L} \in G_{2}^{\kappa_{L}}$

We continue to construct the corresponding solution with the TV-condition. To begin with, draw a curve $C_{L}: u=\omega_{L}-\kappa_{L} \rho^{\gamma}$ from the point $A=A\left(U_{L}\right)$, which intersects the curve $C_{+}^{\kappa_{L}}$ at point $B=B\left(U_{+}\right)$. Through the point $B$, we draw a curve $H_{+}: \rho u=\rho_{+} u_{+}$, which intersects $C_{R}: u=\omega_{L}-\kappa_{R} \rho^{\gamma}$ at points $C$ and $D$, respectively. By Lemma $4.1, H_{+}$is tangent to $C_{R}$ at point $B$. In addition, both points $C$ and $D$ satisfy

$$
\left\{\begin{array}{l}
\rho u=\rho_{+} u_{+}, \\
u+\kappa_{R} \rho^{\gamma}=u_{+}+\kappa_{L} \rho_{+}^{\gamma} .
\end{array}\right.
$$

Combining (5.10) and Lemma 4.2 gives $C=C\left(U_{+}^{0}\right)$ and $D=D\left(U_{+}^{0 \sharp}\right)$, see Fig. 9 .

Similar to the above subcase (I), we may divide the construction into five subcases (B1)(B5), where
(B1): $u_{R}>u_{I}$,
(B2): $u_{+}^{0} \leq u_{R} \leq u_{I}$,
(B3): $\quad u_{+}^{0 \sharp}<u_{R}<u_{+}^{0}$,
(B4): $0 \leq u_{R}<u_{+}^{0 \sharp}$,
(B5): $u_{R}=u_{+}^{0 \sharp}$. 
Figure 9 The Riemann solution at $(\rho, u)$ space for $U_{L}=\left(\rho_{L}, U_{L}, \kappa_{L}\right) \in G_{2}^{K_{L}}$ and $\kappa_{L}>\kappa_{R}$
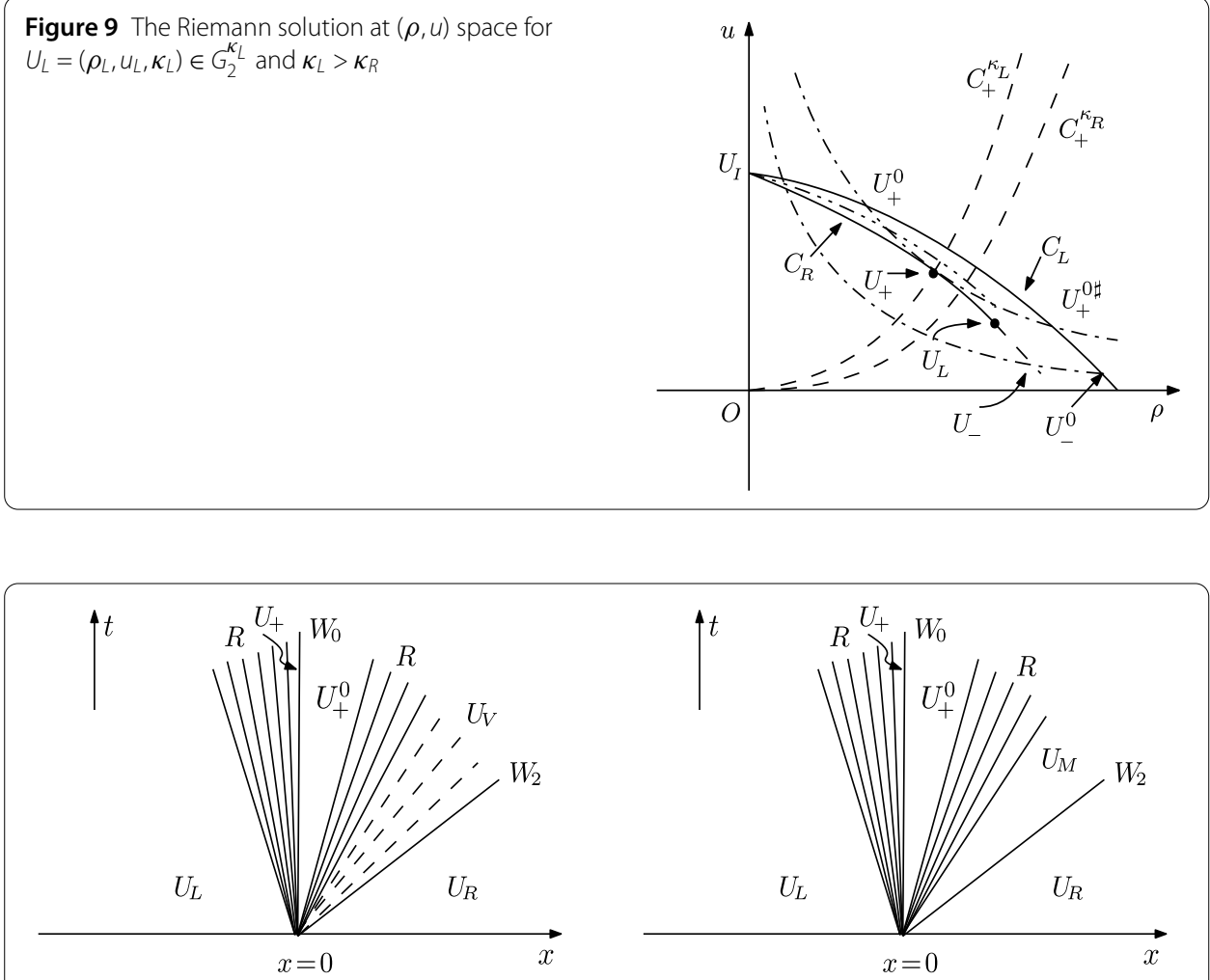

Figure 10 The Riemann solution at $(x, t)$ space for subcases (B1) (left) and (B2) (right), respectively

(B1) $u_{R}>u_{I}$

The Riemann solution begins with a rarefaction wave $R\left(U_{L}, U_{+}\right)$from the state $U_{L}$ to the state $U_{+}=\left(\rho_{+}, u_{+}, \kappa_{L}\right)$, which satisfies

$$
\left\{\begin{array}{l}
u_{L}+\kappa_{L} \rho_{L}^{\gamma}=u+\kappa_{L} \rho^{\gamma}, \\
u=\kappa_{L} \gamma \rho^{\gamma}
\end{array}\right.
$$

Hence there exists a stationary contact wave connecting the state $U_{+}$to state $U_{+}^{0}$ using the $\rho_{1}\left(U_{L}, \kappa_{R}\right)$. The solution is continued with a rarefaction wave $R\left(U_{+}^{0}, U_{I}\right)$, followed by the Vacuum $U_{V}$, where $U_{V}$ is expressed by (5.2). At last, the solution ends up with a contact discontinuity connecting the state $U_{V}$ to $U_{R}$. So, we can construct the Riemann solution as follows:

$$
U_{L}+R\left(U_{L}, U_{+}\right)+U_{+}+W_{0}\left(U_{+}, U_{+}^{0}\right)+U_{+}^{0}+R\left(U_{+}^{0}, U_{I}\right)+U_{V}+W_{2}\left(U_{V}, U_{R}\right)+U_{R}
$$

see Fig. 10.

(B2) $u_{+}^{0} \leq u_{R} \leq u_{I}$.

Based on the above construction, as shown in Fig. 10, we can obtain the Riemann solution

$$
U_{L}+R\left(U_{L}, U_{+}\right)+U_{+}+W_{0}\left(U_{+}, U_{+}^{0}\right)+U_{+}^{0}+R\left(U_{+}^{0}, U_{M}\right)+U_{M}+W_{2}\left(U_{M}, U_{R}\right)+U_{R}
$$


where the state $U_{M}$ is determined by

$$
\left\{\begin{array}{l}
u_{+}^{0}+\kappa_{R}\left(\rho_{+}^{0}\right)^{\gamma}=u+\kappa_{R} \rho^{\gamma}, \\
u=u_{R} .
\end{array}\right.
$$

(B3) $u_{+}^{0 \sharp}<u_{R}<u_{+}^{0}$.

Different from subcase (B2), the rarefaction wave $R\left(U_{+}^{0}, U_{M}\right)$ is presented by a shock wave $S\left(U_{+}^{0}, U_{M}\right)$, where the state $U_{M}$ satisfies (5.12). We can obtain from Fig. 10 the Riemann solution at $(x, t)$ space.

(B4) $0 \leq u_{R}<u_{+}^{0 \sharp}$.

Similar to subcase $(A 4)$, we draw a straight line $u=u_{R}$ from the right state $U_{R}$, which intersects the curve $C_{R}: u=\omega_{L}-\kappa_{R} \rho^{\gamma}$ at some state $U_{-}^{0}$, where $\omega_{L}=u_{L}+p_{L}$ and $U_{-}^{0}=$ $\left(\rho_{-}^{0}, u_{-}^{0}, \kappa_{R}\right)$ satisfies

$$
\left\{\begin{array}{l}
u+\kappa_{R} \rho^{\gamma}=u_{L}+\kappa_{L} \rho_{L}^{\gamma} \\
u=u_{R} .
\end{array}\right.
$$

To continue to construct the solution, we draw a curve $H_{R}: \rho u=\rho_{-}^{0} u_{-}^{0}$ through the state $U_{-}^{0}$ and it intersects the curve $C_{L}: u=\omega_{L}-\kappa_{L} \rho^{\gamma}$ at a state $U_{-}=\left(\rho_{-}, u_{-}, \kappa_{L}\right)$ using $\rho_{2}(\kappa)$, which is determined by

$$
\left\{\begin{array}{l}
\rho u=\rho_{-}^{0} u_{-}^{0}, \\
u+\kappa_{L} \rho^{\gamma}=u_{-}^{0}+\kappa_{R}\left(\rho_{-}^{0}\right)^{\gamma} .
\end{array}\right.
$$

If $u_{-} \geq u_{L}$, there exists a rarefaction wave $R\left(U_{L}, U_{-}\right)$from $U_{L}$ to $U_{-}$. Otherwise, there is a shock wave $S\left(U_{L}, U_{-}\right)$connecting $U_{L}$ to $U_{-}$, see Fig. 9. Thus, the Riemann solution can be described as follows:

$$
U_{L}+W_{1}\left(U_{L}, U_{-}\right)+U_{-}+W_{0}\left(U_{-}, U_{-}^{0}\right)+U_{-}^{0}+W_{2}\left(U_{-}^{0}, U_{R}\right)+U_{R}
$$

We also see Fig. 10.

(B5) $u_{R}=u_{+}^{0 \sharp}$.

As in subcase (A5), we use the monotonicity criterion to consider this subcase and can achieve infinitely many solutions. In fact, from the point $I=I\left(U_{I}\right)$ we draw a curve $C_{\kappa}$ : $u=\omega_{L}-\kappa \rho^{\gamma}$, which intersects the curve $H_{+}$at points $F$ and $G$. By Lemma 4.2, we have $F=F\left(U_{+}^{\kappa}\right)$ and $G=G\left(U_{+}^{\kappa \sharp}\right)$ (see the dashed curve through the point $I$ in Fig. 9). So, there is a stationary contact wave $W_{0}\left(U_{+}, U_{+}^{\kappa}\right)$ connecting $U_{+}$to $U_{+}^{\kappa}=\left(\rho_{+}^{\kappa}, u_{+}^{\kappa}, \kappa\right)$, followed by a zero-speed shock $S_{0}\left(U_{+}^{\kappa}, U_{+}^{\kappa \sharp}\right)$, where $\kappa_{R}<\kappa<\kappa_{L}$. Moreover, we also obtain a stationary contact wave $W_{0}\left(U_{+}^{\kappa \sharp}, U_{+}^{0 \sharp}\right)$ connecting $U_{+}^{\kappa \sharp}$ to $U_{+}^{0 \sharp}$.

(a) If $\kappa_{R}<\kappa<\kappa_{L}$, as shown in Fig. 11, the Riemann solution can expressed by

$$
\begin{aligned}
U_{L} & +R\left(U_{L}, U_{+}\right)+U_{+}+\underbrace{W_{0}\left(U_{+}, U_{+}^{\kappa}\right)+U_{+}^{\kappa}+S_{0}\left(U_{+}^{\kappa}, U_{+}^{\kappa \sharp}\right)+U_{+}^{\kappa \sharp}+W_{0}\left(U_{+}^{\kappa \sharp}, U_{+}^{0 \sharp}\right)}_{x=0} \\
& +U_{+}^{0 \sharp}+W_{2}\left(U_{+}^{0 \sharp}, U_{R}\right)+U_{R} .
\end{aligned}
$$



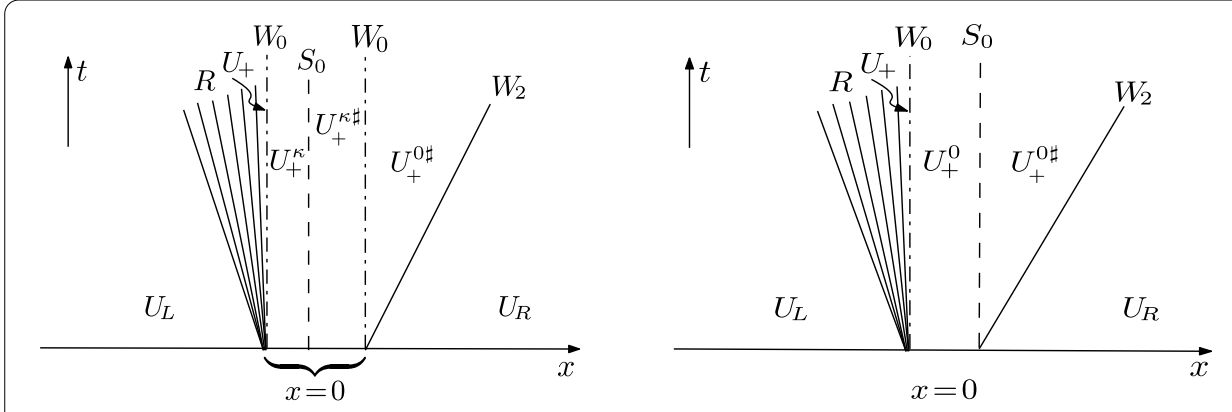

Figure 11 The Riemann solution at ( $x, t)$ space for subcases (B5)-(a) (left) and (B5)-(b) (right), respectively

(b) If $\kappa=\kappa_{R}$, the Riemann solution can be denoted by

$$
\begin{aligned}
U_{L} & +R\left(U_{L}, U_{+}\right)+U_{+}+\underbrace{W_{0}\left(U_{+}, U_{+}^{0}\right)+U_{+}^{0}+S_{0}\left(U_{+}^{0}, U_{+}^{0 \sharp}\right)}_{x=0} \\
& +U_{+}^{0 \sharp}+W_{2}\left(U_{+}^{0 \sharp}, U_{R}\right)+U_{R},
\end{aligned}
$$

which is given in Fig. 11.

Since $\kappa_{R} \leq \kappa<\kappa_{L}$, we easily see the no-uniqueness for the Riemann solution in this subcase.

Under TV-condition (1.6), it follows from Lemma 4.1 that the minimum of $\operatorname{TV}_{W_{0}}(\rho)$ is $\left|\rho_{+}-\rho_{+}^{0}\right|$, which indicates that the Riemann solution is expressed by (5.14). So, we also obtain a unique solution.

(III) $U_{L} \in C_{+}^{\kappa_{L}}$

From the point $A=A\left(U_{L}\right)$, we draw two curves $H_{L}: \rho u=\rho_{L} u_{L}$ and $C_{L}: u=\omega_{L}-\kappa_{L} \rho^{\gamma}$ which intersect the $u$-axis at point $I=I\left(U_{I}\right)$. We again draw a curve $C_{R}: u=\omega_{R}-\kappa_{R} \rho^{\gamma}$ which intersects the $H_{L}$ at two points $B$ and $C$. By Lemmas 4.1 and 4.2, we have $B=B\left(U_{L}^{0}\right)$ and $C=C\left(U_{L}^{0 \sharp}\right)$, see Fig. 1 , where $U_{\alpha}=U_{L}^{0}$ and $U_{\beta}=U_{L}^{0 \sharp}$. As for the Riemann solution in this subcase, we can refer to subcase (II): $U_{L} \in G_{2}^{\kappa_{L}}$.

\subsection{The Riemann solution for the case $\kappa_{L}<\kappa_{R}$}

From the point $A=A\left(U_{L}\right)$, we draw two curves $H_{L}: \rho u=\rho_{L} u_{L}=m_{L}$ and $C_{L}: u=\omega_{L}-$ $\kappa_{L} \rho^{\gamma}$ which intersect the $u$-axis at point $I=I\left(U_{I}\right)$, and again draw a curve $C_{R}: u=\omega_{L}-$ $\kappa_{R} \rho^{\gamma}$, where $\omega_{L}=u_{L}+p_{L}$. Suppose that $C_{R}$ intersects $C_{+}^{\kappa_{R}}$ at state $U_{+}$. And then, we draw a curve $H_{+}: \rho u=\rho_{+} u_{+}=m_{+}$which intersects $C_{L}$ at two states $U_{\alpha}$ and $U_{\beta}$, see Fig. 12. By Lemma 4.1, the $H_{+}$is tangent to $C_{R}$ at state $U_{+}$, and $U_{\alpha}=U_{+}^{0}$ and $U_{\beta}=U_{+}^{0 \sharp}$. We use the curve $H_{+}$to divide the phase plane $\{\rho \geq 0, u \geq 0\}$ into two parts $G_{H}^{L}$ and $G_{H}^{R}$, where

$$
G_{H}^{L}=\left\{(\rho, u) \mid 0 \leq \rho \leq \frac{m_{+}}{u}\right\} \quad \text { and } \quad G_{H}^{R}=\left\{(\rho, u) \mid \rho>\frac{m_{+}}{u}\right\}
$$

We divide this case into three subcases: (I) $U_{L} \in G_{1}^{\kappa_{R}} \cap G_{H}^{L}$, (II) $U_{L} \in G_{2}^{\kappa_{R}} \cup G_{H}^{R}$, and (III) $U_{L}=U_{\alpha}$. In the following, we omit the construction of the Riemann solution at $(x, t)$ space and can obtain the corresponding results as stated above.

(I) $U_{L} \in G_{1}^{\kappa_{R}} \cap G_{H}^{L}$ 
Figure 12 The Riemann solution at $(\rho, u)$ space for $U_{L} \in G_{1}^{K_{R}} \cap G_{H}^{L}$ and $\kappa_{L}<\kappa_{R}$

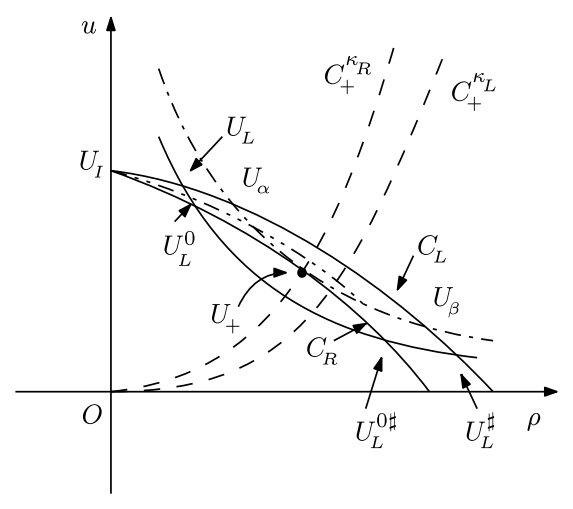

Figure 13 The Riemann solution at $(\rho, u)$ space for $U_{L} \in G_{2}^{\kappa_{R}} \cap G_{H}^{R}$ and $\kappa_{L}<\kappa_{R}$

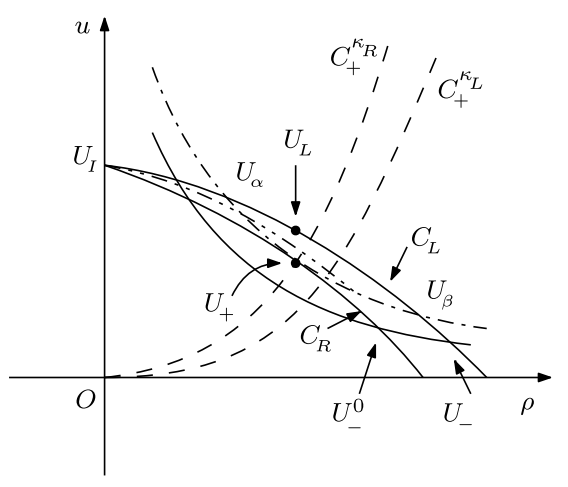

Figure 12 shows that the Riemann solution in this subcase is similar to that in subcase (I) of Sect. 5.2, and here omit the details.

(II) $U_{L} \in G_{2}^{\kappa_{R}} \cup G_{H}^{R}$

By the location of the right state $U_{R}$, we divide this into $(\mathrm{C} 1) u_{R} \geq u_{+}$and $(\mathrm{C} 2) 0 \leq u_{R}<$ $u_{+}$, see Fig. 13 .

(C1) $u_{R} \geq u_{+}$.

The Riemann solution begins with $W_{1}\left(U_{L}, U_{\beta}\right)$ from $U_{L}$ to $U_{\beta}$. If $u_{\beta}<u_{L}<u_{\alpha}$, then one has $\sigma\left(U_{L}, U_{\alpha}\right)<0$ by Lemma 3.1 and $W_{1}\left(U_{L}, U_{\beta}\right)$ is a shock wave $S\left(U_{L}, U_{\beta}\right)$. Otherwise, there exists a rarefaction wave $R\left(U_{L}, U_{\beta}\right)$ connecting $U_{L}$ and $U_{\beta}$. The $W_{1}\left(U_{L}, U_{\beta}\right)$ is followed by a stationary contact wave $W_{0}\left(U_{\beta}, U_{+}\right)$connecting $U_{\beta}$ and $U_{+}$. And then, we refer to subcases (A1) and (A2) and construct the corresponding Riemann solution.

(C2) $0 \leq u_{R}<u_{+}$.

As for this subcase, we see subcase (B4).

(III) $U_{L}=U_{\alpha}$

We can divide this into two subcases (D1) $u_{R} \geq u_{+}$and (D2) $0 \leq u_{R}<u_{+}$.

If we apply the monotonicity criterion to the two subcases, then similar to two subcases (A5) and (B5), through the point $I=I\left(U_{I}\right)$ we draw a curve $C_{\kappa}: u=\omega_{L}-\kappa \rho^{\gamma}$, which intersects the curve $H_{+}$at points $F$ and $G$, where $\kappa_{L}<\kappa<\kappa_{L}$. By Lemma 4.2, we have $F=F\left(U_{L}^{\kappa}\right)$ and $G=G\left(U_{L}^{\kappa \sharp}\right)$ (see the dashed curve through the point $I$ in Fig. 13).

As for subcases (D1), we need only to consider the case from $U_{L}$ to $U_{+}$. 
To begin with, there is a stationary contact wave $W_{0}\left(U_{L}, U_{L}^{\kappa}\right)$ from $U_{L}=U_{\alpha}$ to $U_{L}^{\kappa}=$ $\left(\rho_{L}^{\kappa}, u_{L}^{\kappa}, \kappa\right)$, followed by a zero-speed shock $S_{0}\left(U_{L}^{\kappa}, U_{L}^{\kappa \sharp}\right)$, where $\kappa_{R}<\kappa<\kappa_{L}$. The solution is continued with a stationary contact wave $\mathcal{W}_{0}\left(U_{L}^{\kappa \sharp}, U_{+}\right)$, see Fig. 13.

If $\kappa=\kappa_{L}$, then there is a zero-speed shock $S_{0}\left(U_{L}, U_{\beta}\right)$ connecting $U_{L}$ and $U_{\beta}$, where $U_{\beta}=U_{+}^{0 \sharp}$. The $S_{0}\left(U_{L}, U_{\beta}\right)$ is followed by a stationary contact wave $W_{0}\left(U_{+}^{0 \sharp}, U_{+}\right)$from $U_{+}^{0 \sharp}$ to $U_{+}$.

If $\kappa=\kappa_{R}$, there is only one stationary contact wave $\mathcal{W}_{0}\left(U_{L}, U_{+}\right)$from $U_{L}$ to $U_{+}$.

Obviously, there are infinitely many solutions for this subcase. When we impose the TVcondition, it is easy to see that only the stationary contact wave $\mathcal{W}_{0}\left(U_{L}, U_{+}\right)$is allowed.

In addition, we can refer to subcase (A4) and obtain the corresponding solution for the subcase (D2): $0 \leq u_{R}<u_{+}$.

Thus, we use the TV-condition to consider the Riemann problem case by case and achieve the uniqueness of Riemann solution for each subcase. So, we complete the proof of Theorem 1.1.

\section{Conclusions}

Through discussions in this paper, we see that there may exist infinitely many solutions to the Aw-Rascle model for certain Riemann data. So, we propose the so-called TVcondition, which allows us to single out a unique solution among possibly infinitely many solutions. It is a meaningful and valuable exploration to study the resonance behavior of hyperbolic systems, even though until now we cannot confirm that the unique solution thus selected is a physically relevant one. We plan to address this problem and generalize a similar condition to study other systems in $[1,11,27,28]$ in future work.

\section{Acknowledgements}

The authors would like to express their sincere gratitude to the anonymous referees and the editor for many friendly and helpful suggestions, which led to a great deal of improvement of the original manuscript.

\section{Funding}

The work of Y.B. Hu was partially supported by the National Science Foundation of China under Grants 11301128 and 11571088, and Zhejiang Provincial Natural Science Foundation under Grant LY17A010019. The work of G.D. Wang was partially supported by the Science Key Project of Education Department of Anhui Province under Grants KJ2018A0517, KJ2019A0782, KJ2019A0786 and the Scientific Research Foundation of Anhui Jianzhu University under Grants JZ192050 2018QD36, 2016QD110.

\section{Competing interests}

The authors declare that they have no competing interests.

Authors' contributions

All authors carried out the proofs and conceived of the study. All authors read and approved the final manuscript.

\section{Author details}

${ }^{1}$ Department of Mathematics, Hangzhou Normal University, Hangzhou, P.R. China. ${ }^{2}$ School of Mathematics and Physics, Anhui Jianzhu University, Hefei, P.R. China.

\section{Publisher's Note}

Springer Nature remains neutral with regard to jurisdictional claims in published maps and institutional affiliations.

Received: 20 February 2019 Accepted: 8 January 2020 Published online: 07 February 2020

\section{References}

1. Ambroso, A., Chalons, C., Coquel, F., Godlewski, E., Galié, T.: Interface model coupling via prescribed local flux balance. ESAIM: Math. Model. Numer. Anal. 48, 895-918 (2014)

2. Ambroso, A., Chalons, C., Coquel, F., Godlewski, E., Lagoutière, F., Raviart, P.A., Seguin, N.: A relaxation method for the coupling of systems of conservation laws. In: Hyperbolic Problem: Theory, Numerics, Applications, pp. 947-954. Springer, Berlin (2008) 
3. Ambroso, A., Chalons, C., Coquel, F., Godlewski, E., Lagoutière, F., Raviart, P.A., Seguin, N.: Relaxation methods and coupling procedure. Int. J. Numer. Methods Fluids 56, 1123-1129 (2008)

4. Ambroso, A., Chalons, C., Coquel, F., Godlewski, E., Lagoutière, F., Raviart, P.A., Seguin, N.: Coupling of general Lagrangian systems. Math. Comput. 77, 909-941 (2008)

5. Andreianov, B., Karlsen, K.H., Risebro, H.N.: A theory of $L^{1}$-dissipative solvers for scalar conservation laws with discontinuous flux. Arch. Ration. Mech. Anal. 201, 27-86 (2011)

6. Andrianov, N., Warnecke, G.: On the solution to the Riemann problem for the compressible duct flow. SIAM J. Appl. Math. 64, 878-901 (2004)

7. Aw, A., Rascle, M.: Resurrection of "second order" models of traffic flow. SIAM J. Appl. Math. 60, 916-938 (2000)

8. Boutin, B., Coquel, F., LeFloch, P.G.: Coupling techniques for nonlinear hyperbolic equations. III. The well-balanced approximation of thick interfaces. SIAM J. Numer. Anal. 51, 1108-1133 (2013)

9. Boutin, B., Coquel, F., LeFloch, P.G.: Coupling techniques for nonlinear hyperbolic equations. IV. Well-balanced schemes for scalar multi-dimensional and multi-component laws. Math. Comput. 84, 1663-1702 (2015)

10. Chalons, C., Raviart, P.A., Seguin, N.: The interface coupling of the gas dynamics equations. Q. Appl. Math. 66, 659-705 (2008)

11. Chatzarakis, G.E., Li, T.: Oscillation criteria for delay and advanced differential equations with nonmonotone arguments. Complexity 2018, Article ID 8237634 (2018)

12. Coquel, F., Godlewski, E., Haddaoui, K., Marmignon, C., Renac, F.: Choice of measure source terms in interface coupling for a model problem in gas dynamics. Math. Comput. 85, 2305-2339 (2016)

13. Cuong, D.H., Thanh, M.D.: A Godunov-type scheme for the isentropic model of a fluid flow in a nozzle with variable cross-section. Appl. Math. Comput. 256, 602-629 (2015)

14. Dal Maso, G., Lefloch, P.G., Murat, F.: Definition and weak stability of nonconservative products. J. Math. Pures Appl. 74, 483-548 (1995)

15. Gimse, T., Risebro, N.H.: Riemann problems with a discontinuous flux function. In: Engquist, B., Gustafsson, B. (eds.) Proc. Third Internat. Conf. on Hyperbolic Problems. Theory, Numerical Method and Applications, pp. 488-502. Studentlitteratur/Chartwell-Bratt, Lund-Bromley (1991)

16. Gimse, T., Risebro, N.H.: Solution of the Cauchy problem for a conservation law with a discontinuous flux function. SIAM J. Math. Anal. 23(3), 635-648 (1992)

17. Goatin, P., LeFloch, P.G.: The Riemann problem for a class of resonant hyperbolic systems of balance laws. Ann. Inst. Henri Poincaré, Anal. Non Linéaire 21, 881-902 (2004)

18. Han, E., Hantke, M., Warnecke, G.: Exact Riemann solutions to compressible Euler equations in ducts with discontinuous cross section. J. Hyperbolic Differ. Equ. 9, 1-47 (2012)

19. Han, E., Hantke, M., Warnecke, G.: Criteria for nonuniqueness of Riemann solutions to compressible duct flows. J. Appl. Math. Mech. 93, 465-475 (2013)

20. Han, E., Hantke, M., Warnecke, G.: Exact Riemann solutions to shallow water equations. Q. Appl. Math. 72, 407-453 (2014)

21. Hong, J.M., Hsu, C.H., Huang, B.C.: Existence and uniqueness of generalized stationary waves for viscous gas flow through a nozzle with discontinuous cross section. J. Differ. Equ. 253, 1088-1110 (2012)

22. Hong, J.M., Hsu, C.H., Lin, Y.C., Liu, W.: Linear stability of the sub-to-super inviscid transonic stationary wave for gas flow in a nozzle of varying area. J. Differ. Equ. 254, 1957-1976 (2013)

23. Isaacson, E., Temple, B.: Nonlinear resonance in systems of conservation laws. SIAM J. Appl. Math. 52, 1260-1278 (1992)

24. Isaacson, E., Temple, B.: Convergence of the $2 \times 2$ Godunov method for a general resonant nonlinear balance law. SIAM J. Appl. Math. 55, 625-640 (1995)

25. Jiang, W.F., Wang, Z.: Developing an Aw-Rascle model of traffic flow. J. Eng. Math. 97, 135-146 (2016)

26. Jin, S., Wen, X.: Two interface type numerical methods for computing hyperbolic systems with geometrical source terms having concentrations. SIAM J. Sci. Comput. 26, 2079-2101 (2005)

27. LeFloch, P.G., Thanh, M.D.: The Riemann problem for fluid flows in a nozzle with discontinuous cross-section. Commun. Math. Sci. 1, 763-797 (2003)

28. LeFloch, P.G., Thanh, M.D.: The Riemann problem for the shallow water equations with discontinuous topography. Commun. Math. Sci. 5, 865-885 (2007)

29. LeFloch, P.G., Thanh, M.D.: A Godunov-type method for the shallow water equations with discontinuous topography in the resonant regime. J. Comput. Phys. 230, 7631-7660 (2011)

30. Liu, T.P.: Quasilinear hyperbolic systems. Commun. Math. Phys. 68, 141-172 (1979)

31. Liu, T.P.: Resonance for quasilinear hyperbolic equation. Bull. Am. Math. Soc. 6, 463-465 (1982)

32. Marchesin, D., Paes-Leme, P.J.: A Riemann problem in gas dynamics with bifurcation. Comput. Math. Appl. 12A, 433-455 (1986)

33. Shen, W: Global Riemann solvers for several $3 \times 3$ systems of conservation laws with degeneracies. Math. Models Methods Appl. Sci. 28(8), 1599-1626 (2018)

34. Sun, M.: Interactions of elementary waves for Aw-Rascle model. SIAM J. Appl. Math. 69, 1542-1558 (2009)

35. Temple, B.: Global solution of the Cauchy problem for a class of $2 \times 2$ nonstrictly hyperbolic conservation laws. Adv. Appl. Math. 3, 335-375 (1982)

36. Temple, B.: Systems of conservation laws with invariant submanifolds. Trans. Am. Math. Soc. 280, 781-795 (1983)

37. Thanh, M.D.: The Riemann problem for a nonisentropic fluid in a nozzle with discontinuous cross-sectional area. SIAM J. Appl. Math. 69, 1501-1519 (2009)

38. Thanh, M.D.: Properties of the wave curves in the shallow water equations with discontinuous topography. Bull. Malays. Math. Sci. Soc. 39, 305-337 (2016)

39. Thanh, M.D., Kröner, D.: Testing improvements of a well-balanced method for the model of a fluid in a nozzle with variable cross-section. Taiwan. J. Math. 18, 1739-1757 (2014) 